\title{
UNIPOTENT REPRESENTATIONS AND REDUCTIVE DUAL PAIRS OVER FINITE FIELDS
}

\author{
JEFFREY ADAMS AND ALLEN MOY
}

\begin{abstract}
Consider the representation correspondence for a reductive dual pair $\left(G_{1}, G_{2}\right)$ over a finite field. We consider the question of how the correspondence behaves for unipotent representations. In the special case of cuspidal unipotent representations, and a certain fundamental situation, that of "first occurrence", the representation correspondence takes a cuspidal unipotent representation of $G_{1}$ to one of $G_{2}$. This should serve as a fundamental case in studying the correspondence in general over both finite and local fields.
\end{abstract}

Let $\left(G, G^{\prime}\right)$ be a reductive dual pair over a field $\mathbf{F}$, and consider the representation correspondence coming from the oscillator representation. Thus we have a correspondence, written $\pi \rightarrow \pi^{\prime}$, from irreducible representations of $G$ to those of $G^{\prime}$. It is of interest to compute explicitly this correspondence. While we ultimately are interested in this problem for $\mathbf{F}$ a field of characteristic zero, we consider the problem here for $\mathbf{F}$ a finite field (cf. [H1]). Srinivasan [S] has given a partial but important solution to this problem in terms of a formula for the decomposition of the oscillator representation into a sum of Deligne-Lusztig representations.

Most schemes for explicitly describing the representation correspondence involve a reduction to some type of fundamental case. For $\mathbf{F}$ a $p$-adic field this may be supercuspidal representations (cf. [K, MVW]). For $\mathbf{F}$ the real numbers the obvious analogue of the supercuspidal representations is the discrete series. They do not however form a large enough set. There is some evidence that one needs to include unipotent representations, although this notion has yet to be completely defined (cf. $[\mathrm{A}, \mathrm{V}])$.

For $\mathbf{F}$ a finite field it seems likely that the representations which are both unipotent and cuspidal should play a special role, being fundamental in both of these senses. Such representations are quite rare. For classical groups they exist only for unitary, symplectic, and orthogonal groups of certain degrees, and in this case are unique (cf. [L2], also Theorem 5.1). These groups occur within reductive dual pairs of type I. Theorem 3.6 states that under suitable conditions the cuspidal unipotent representations of these groups correspond under the representation correspondence.

Received by the editors September 8, 1991.

1980 Mathematics Subject Classification (1985 Revision). Primary 20C33; Secondary 22E46.

Partially supported by NSF grants DMS-8802586 and DMS-8701429. 
More precisely, we show that cuspidal unipotent representations correspond in the case of first occurrence (cf. [K]). Thus for a fixed group $G$, and an irreducible representation $\pi$ of $G$, we consider the smallest group $G^{\prime}$ of a given Witt class such that $\pi$ occurs in the representation correspondence for this dual pair. In Theorem 3.6 we show, under the hypothesis of first occurrence, that if $\pi$ is a cuspidal representation, and $\pi \rightarrow \pi^{\prime}$, then $\pi^{\prime}$ is also a cuspidal unipotent representation. The proof is very simple. It follows easily from Srinivasan's formula that the oscillator correspondence takes unipotent representations to unipotent representations. This is Theorem 3.5. Also, by carrying over the argument of $[\mathrm{K}]$, we show in Theorem 2.2 that cuspidal representations correspond in the case of first occurrence.

It remains to compute in which dimensions the cases of first occurrence occur, and the actual correspondence. We use a different method for this in each of two cases. In the case of unitary groups it is possible to invert Srinivasan's formula for the oscillator representation, which is in terms of $R_{T}(\theta)$ 's, to obtain a result on irreducible representations. We carry this out in $\S 4$. In fact, for unitary groups we show directly, without recourse to Theorem 3.6, that cuspidal unipotent representations correspond as stated. In $\S 5$ we prove the result for orthogonal-symplectic pairs using some simple results of [H1]. Here, Theorem 3.6 and the sparsity of cupsidal unipotent representations impose some strong constraints on the correspondence. This fact together with some weak information on where representations can occur is enough to pin down the result completely.

Several examples of this have been noted by other authors. The representation of $S p(4)$ corresponding to the sgn representation of $\mathrm{O}^{-}(2)$ is Srinivasan's $\theta_{10}$. For all $n$ the disconnectedness of $O^{ \pm}(n)$ continues to play a critical role. Another example is the trivial representation of $U(1)$ going to the cuspidal unipotent representation of $U(3)$ [H1].

In this paper we consider only even orthogonal groups, in part due to this restriction in [S] (cf. 3.11). In the case of odd orthogonal groups, the analogue of Theorem 3.5 is false. This is seen by considering the pair $(S p(2), O(1))$. The correspondence given by the oscillator representation of $S p(2)$ does not preserve unipotent representations. We hope to return to this matter in a later paper.

We thank S. Rallis for pointing out the reference [S], and W. Feit, R. Howe, and S. Kudla for helpful discussions. We also thank J. Michel for discovering a minor error in our original proof of Theorem 3.5(2), and for supplying us with information needed to repair the proof.

\section{2}

Let $\mathbf{F}$ be a finite field of odd order, $\mathbf{K}$ a finite extension of $\mathbf{F}$ and $\emptyset$ an involution of $\mathbf{K}$ for which $\mathbf{F}$ is the fixed field. This means either (i) $\mathbf{K}$ is equal to $\mathbf{F}$ and $\downarrow$ is the trivial involution or (ii) $\mathbf{K}$ is a quadratic extension of $\mathbf{F}$ and $b$ is the Galois involution. We recall the classification of finite dimensional nondegenerate $\downarrow$-Hermitian and -skew-Hermitian forms.

Case (i) $(\mathbf{F}=\mathbf{K})$. A form. There are two such forms in each positive dimension, classified by the discriminant. In particular, the two dimensional split form has discriminant 
-1 . In dimension zero, we use the convention that there is one form and that its discriminant is 1 . In the case of odd dimension the two classes of forms can be related to one another by multiplying the forms by a nonsquare element in $\mathbf{F}$. This means the isometry groups of the two classes of orthogonal forms in odd dimension are naturally isomorphic. In the even dimensional case the isometry groups of the two classes of forms are not isomorphic. We use the convention that $O^{+}(2 m)$ (resp. $O^{-}(2 m)$ ) denotes the isometry group of the split (resp. nonsplit) form in dimension $2 m$.

A $b$-skew-Hermitian form is a symplectic form. The dimension, which is of course even, say $2 m$, completely specfies the form. The isometry group is the symplectic group $S p(2 m)$.

Case (ii) $([\mathbf{K}: \mathbf{F}]=2)$. Any two nondegenerate Hermitian forms in a given dimension are equivalent. Furthermore if $($,$) is a Hermitian form and \tau$ is a nonzero element of $\mathbf{K}$ such that $\tau^{\natural}=-\tau$ then $\langle\rangle=,\tau \cdot($, ) is skew-Hermitian. This means there is also, up to equivalence, one nondegenerate skew-Fiermitian form in each dimension. The unitary group $U(n)$ is then the isometry group of either an Hermitian or skew-Hermitian form in dimension $n$.

Suppose now $V$ (resp. $W$ ) is a finite dimensional vector space over $\mathbf{K}$ with a nondegenerate form $($,$) (resp. \langle\rangle$,$) , one \sharp$-skew-Hermitian and one q-Hermitian. Let $G$ (resp. $G^{\prime}$ ) be the isometry group of $($,$\left.) (resp. \langle\rangle,\right)$. The space $\mathbf{W}=V \otimes_{\mathbf{K}} W$ carries the symplectic form $\langle\langle\rangle\rangle=,\operatorname{tr}_{\mathbf{K} / \mathbf{F}}\left((,) \otimes_{\mathbf{K}}\langle\rangle,\right)$ and $\left(G, G^{\prime}\right)$ embeds as a reductive dual pair in $S p(\mathbf{W})$. The possiblities for $\left(G, G^{\prime}\right)$ are $(U(m), U(n)),\left(S p(2 m), O^{ \pm}(n)\right)$, and $\left(O^{ \pm}(n), S p(2 m)\right)$.

By definition a Witt tower is a maximal sequence of spaces $\left\{V_{k}\right\}_{k}$, each with an Hermitian form such that $V_{k+1}$ is the direct sum of $V_{k}$ and a hyperbolic $\mathbf{K}$-plane. The index $k$ runs over either $\mathbf{N}$ or $\mathbf{N} \cup\{0\}$ as explained below. Let $G_{k}=G\left(V_{k}\right)$ be the isometry group of $V_{k}$. We refer to $\left\{G_{k}\right\}_{k}$ as a Witt tower of groups. If $G$ is the isometry group of an Hermitian form on a vector space $V$, we embed $G$ in a Witt tower of groups as follows.

Case (i) $(\mathbf{F}=\mathbf{K})$. Suppose $\natural$ is symmetric. There is a Witt tower of split even dimensional forms, with $G_{k} \approx O^{+}(2 k)$. Here we let $k$ run over $\mathbf{N} \cup\{0\}$, with $V_{0}$ taken to be the 0 -dimensional space, and $O^{+}(0)$ the trivial group. We have a Witt tower of nonsplit orthogonal groups $G_{k} \approx O^{-}(2 k) \quad(k \in \mathbf{N})$. Similarly we obtain two Witt towers of odd orthogonal groups $G_{k} \approx O^{ \pm}(2 k-1) \quad(k \in \mathbf{N})$.

There is one symplectic Witt tower, with $G_{k} \approx S p(2 k) \quad(k \in \mathbf{N} \cup\{0\})$, where $S p(0)$ is the trivial group.

Case (ii) $([\mathbf{K}: \mathbf{F}]=2)$. There are two Hermitian Witt towers, $G_{k} \approx U(2 k)$ $(k \in \mathbf{N} \cup\{0\})$ and $G_{k} \approx U(2 k-1) \quad(k \in \mathbf{N})$. As mentioned above the skewHermitian case is isomorphic.

Suppose $\left(G, G^{\prime}\right)$ is a reductive dual pair in $S p(\mathbf{W})$ constructed as above. Embed $G$ (resp. $\left.G^{\prime}\right)$ in a Witt tower of groups $G_{k}$ (resp. $G_{k}^{\prime}$ ) such that $G_{m} \approx$ $G$ (resp. $\left.G_{n}^{\prime} \approx G^{\prime}\right)$. Then each $\left(G_{k}, G_{l}^{\prime}\right)$ is a reductive dual pair in some symplectic group. Fix an additive character $\chi$ of $\mathbf{F}$, and let $\omega_{k, l}$ denote the corresponding oscillator representation restricted to $\left(G_{k}, G_{l}^{\prime}\right)$. This defines the representation correspondence between $G_{k}$ and $G_{l}^{\prime}$. In particular, $\omega_{m, n}$ defines the representation correspondence between $G$ and $G^{\prime}$. 
Theorem 2.2. Suppose $\pi$ is a cuspidal representation of $G$, and

(1) $\pi \leftrightarrow \pi^{\prime}$ in the dual pair correspondence for $G \times G_{n}^{\prime}$,

(2) $\pi$ does not occur in the correspondence for $G \times G_{k}^{\prime}$ for any $k<n$.

Then $\pi^{\prime}$ is cuspidal.

We refer to the occurrence of a given representation $\pi$ of $\mathrm{G}$ with $\mathrm{n}$ minimal as in the statement of the theorem, as the case of first occurrence.

The proof is distilled from Theorems 2.5 and 2.8 of [K]. We begin by establishing in the finite field case a result of Kudla on the relationship between the oscillator representation and the Jacquet functor. Let $X$ be an isotropic subspace of $W$ of dimension $j$, let $Y$ be an isotropic complement to $X$, and let $W_{0}$ be an orthogonal complement of $X \oplus Y$ in $W$. Associated to the decomposition $W=X \oplus W_{0} \oplus Y$ is a maximal parabolic subgroup $P^{\prime}$ of $G^{\prime}$. Let $P^{\prime}=M^{\prime} N^{\prime}$ be a Langlands decomposition of $P^{\prime}$ where $M^{\prime} \simeq G L(X, \mathbf{K}) \times G_{n-j}^{\prime}$.

The Jacquet module $R\left(M^{\prime}, \omega_{m, n}\right)=\left(\omega_{m, n}\right)_{N^{\prime}}$ is a representation of $G \times$ $M^{\prime}$, which by restriction we consider as a representation of $G \times G_{n-j}^{\prime}$. Denote by $R\left(M^{\prime}, \omega_{m, n}\right)_{\text {cusp }}$ the cuspidal part of the restriction of $\left(\omega_{m, n}\right)_{N^{\prime}}$ to $G$.

\section{Proposition 2.3.}

$$
R\left(M^{\prime}, \omega_{m, n}\right)_{\text {cusp }} \simeq \omega_{m, n-j}
$$

Proof. We use a mixed model [H1, H2] of $\omega_{m, n}$. We have the decomposition

$$
\mathbf{W}=(V \otimes X) \oplus\left(V \otimes W_{0}\right) \oplus(V \otimes Y) .
$$

The space of $\omega_{m, n}$ may be taken to be $L^{2}(V \otimes X, S)$, where $S$ is the space of $\omega_{m, n-j}$. Now the center $Z\left(N^{\prime}\right)$ of $N^{\prime}$ is contained in the set of symmetric bilinear forms on $V \otimes X$, via $z \rightarrow \beta_{z}\left(\right.$, ), where $\beta_{z}(x, y)=\langle\langle z x, y\rangle\rangle$. From the fact that $Z\left(N^{\prime}\right)$ is a subgroup of $G^{\prime}(X \oplus Y)$ we see that the action of $Z\left(N^{\prime}\right)$ is given by

$$
\omega_{m, n}(z)(\phi)(x)=\chi\left(\frac{1}{2} \operatorname{tr}_{K / F} \beta_{z}(x, x)\right) \phi(x) \quad\left(z \in Z\left(N^{\prime}\right), x \in V \otimes X\right) .
$$

Write

$$
V \otimes X \simeq \overbrace{V \oplus V \oplus \cdots \oplus V}^{j \text { times }}
$$

corresponding to which for $x \in V \otimes X$ we write $x=\left(x_{1}, x_{2}, \ldots, x_{j}\right)$. Let

$$
X^{0}=\left\{x \mid\left\langle x_{i}, x_{k}\right\rangle=0 \text { for all } i, k\right\},
$$

and let $X_{i}^{0}=\left\{x \in X^{0} \mid \operatorname{dim}\left(\operatorname{span}\left\{x_{1}, x_{2}, \ldots, x_{j}\right\}\right)=i\right\}$. Then it is straightforward to see

$$
\left(\omega_{m, n}\right)_{Z\left(N^{\prime}\right)} \simeq L^{2}\left(X^{0}, S\right),
$$

and this space is equal to

$$
\sum_{i=0}^{\min (j, m)} L^{2}\left(X_{i}^{0}, S\right) .
$$

Since the stabilizer in $G$ of $x \in X_{i}^{0}$ is contained in a parabolic subgroup unless $i=0$, it follows that $\left.L^{2}\left(X_{i}^{0}, S\right)\right|_{G}$ has no cuspidal summands unless $i=0$. Thus we have that the $G$-cuspidal component of $\left(\omega_{m, n}\right)_{Z\left(N^{\prime}\right)}$ is $L^{2}\left(X_{0}^{0}, S\right) \simeq S$. 
Finally there is a complement to $Z\left(N^{\prime}\right)$ in $N^{\prime}$ which acts trivially on $\left.L^{2}\left(X_{i}^{0}, S\right)\right|_{G}$. Thus the cuspidal component of $\left(\omega_{m, n}\right)_{N^{\prime}}$ also is isomorphic to $\mathrm{S} \simeq \omega_{m, n-j}$. This completes the proof of the proposition.

Proof of Theorem 2.2. We argue by contradiction. Suppose that $\pi^{\prime}$ is not cuspidal. Then there exists a maximal parabolic subgroup $P^{\prime}=M^{\prime} N^{\prime}$ as above, and a representation $\sigma^{\prime} \dot{\otimes} \tau^{\prime}$ of $M^{\prime}$ such that

$$
\pi^{\prime} \subset \operatorname{Ind}_{P^{\prime}}^{G^{\prime}}\left(\sigma^{\prime} \check{\otimes} \tau^{\prime} \check{\otimes} 1\right)
$$

Thus

$$
\begin{aligned}
0 & \neq \operatorname{dim}\left(\operatorname{Hom}_{G \times G_{n}^{\prime}}\left(\omega_{m, n}, \pi \check{\otimes} \pi^{\prime}\right)\right) \\
& \leq \operatorname{dim}\left(\operatorname{Hom}_{G \times G_{n}^{\prime}}\left(\omega_{m, n}, \pi \check{\otimes} \operatorname{Ind}_{P^{\prime}}^{G^{\prime}}\left(\sigma^{\prime} \check{\otimes} \tau^{\prime} \check{\otimes} 1\right)\right)\right) \\
& =\operatorname{dim}\left(\operatorname{Hom}_{G \times M^{\prime}}\left(R\left(M^{\prime}, \omega_{m, n}\right), \pi \check{\otimes} \sigma^{\prime} \check{\otimes} \tau^{\prime}\right)\right) \\
& \leq \operatorname{dim}\left(\operatorname{Hom}_{G \times G_{n-j}^{\prime}}\left(\left.R\left(M^{\prime}, \omega_{m, n}\right)\right|_{G \times G_{n-j}^{\prime}}, \pi \check{\otimes} \tau^{\prime}\right)\right) \\
& =\operatorname{dim}\left(\operatorname{Hom}_{G \times G_{n-j}^{\prime}}\left(R\left(M^{\prime}, \omega_{m, n}\right)_{\text {cusp }}, \pi \check{\otimes} \tau^{\prime}\right)\right) \\
& =\operatorname{dim}\left(\operatorname{Hom}_{G \times G_{n-j}^{\prime}}\left(\omega_{m, n-j}, \pi \check{\otimes} \tau^{\prime}\right)\right) \\
& =0 .
\end{aligned}
$$

This is a contradiction.

Fix an algebraic closure $\overline{\mathbf{F}}$ of $\mathbf{F}$ and let $\mathbf{G}$ be the $\overline{\mathbf{F}}$ points of a connected reductive group defined over $\mathbf{F}$. Denote by $F$ the Frobenius map. The group of $\mathbf{F}$-rational points of $\mathbf{G}$ is $G=\mathbf{G}^{F}$. If $\mathbf{T}$ is a maximal torus of $\mathbf{G}$ defined over $\mathbf{F}$ and $\theta$ is a character of $T=\mathbf{T}^{F}$, Deligne and Lusztig have defined [DL] a virtual representation $R_{T}^{G}(\theta)$ of $G$.

We recall the classification of maximal tori defined over over $\mathbf{F}$. Fix a maximal torus $\mathbf{T}_{0}$ in $\mathbf{G}$ which is defined over $\mathbf{F}$ and which is maximally $\mathbf{F}$-split. Let $\mathbf{W}$ be the Weyl group of $\mathbf{T}_{0}$. The Frobenius $F$ acts on $\mathbf{W}$ and two elements $g, h \in \mathbf{W}$ are said to be $F$-conjugate if $g=x \cdot h \cdot F(x)^{-1}$ for some $x \in \mathbf{W}$. The $F$-conjugacy classes in $\mathbf{W}$ parametrize the maximal tori of $\mathbf{G}$ defined over $\mathbf{F}$. If $\mathbf{G}$ is split over $\mathbf{F}$, the Frobenius action on $\mathbf{W}$ is trivial and an $F$-conjugacy class is just a conjugacy class.

An irreducible representation of $G$ is said to be unipotent if it occurs as a constituent of some $R_{T}^{G}(1)$. In the case of an orthogonal group $O^{\epsilon}$, we define a unipotent representation to be a constituent of $\operatorname{Ind}_{S O^{*}}^{\sigma}(\pi)$, for $\pi$ a unipotent representation of $S O^{\epsilon}$.

Suppose $\mathbf{P}$ is a parabolic subgroup of $\mathbf{G}$ such that $\mathbf{P}$ contains a Levi subgroup $\mathbf{L}$ which is defined over $\mathbf{F}$. The group $\mathbf{P}$ may or may not be defined over F. If $\theta$ is a character of $L=\mathbf{L}^{F}$, Deligne and Lusztig have defined a virtual representation $R_{L}^{G}(\theta)$ of $G$ by

$$
R_{L}^{G}(\theta)=\sum_{(T) \subset L} \frac{1}{\left|W_{L}(T)\right|} R_{T}^{G}(\theta) .
$$

Here, the sum is over representatives for the maximal tori in $L$, and $W_{L}(T)$ is the Weyl group of $T$ in $L$. The next result follows immediately from the orthogonality relations satisfied by the $R_{T}^{G}(\theta)$ 's. 


\section{Theorem 3.1.}

(1) A unipotent representation occurs in $R_{L}^{G}(\theta)$ only if $\theta=1$.

(2) $R_{L}^{G}(1)$ consists solely of unipotent representations.

We discuss the unitary case in dimension $n$, since it will be needed later. We take $\mathbf{G}=G L(n, \overline{\mathbf{F}})$. To describe the Frobenius action, let $J \in \mathbf{G}$ be the matrix which is one on the antidiagonal and zero elsewhere. Then

$$
F\left(x_{i, j}\right)=J \cdot\left(x_{j, i}^{q}\right) \cdot J .
$$

The set $\mathbf{T}_{0}$ of diagonal matrices in $\mathbf{G}$ is a maximally $\mathbf{F}$-split maximal torus in $\mathbf{G}$. The normalizer of $\mathbf{T}_{0}$ in $\mathbf{G}$ consists of the monomial matrices and $\mathbf{W}$ can be identified with the symmetric group $S_{n}$. The image $w_{J}$ of $J$ in $\mathbf{W}$ corresponds to the longest element, and the Frobenius action on $\mathbf{W}$ is given by

$$
F(w)=w_{J} \cdot w \cdot w_{J}
$$

This means there is a simple relationship between $F$-conjugacy classes and ordinary conjugacy classes. One type of class is obtained from the other by multiplication on the right by $w_{J}$.

We review the classification of unipotent representations of $U(n)$ in [LS] which we shall need for later calculations. As mentioned above, an $F$-conjugacy class $\mathscr{C}_{F}$ of $W=S_{n}$ is obtained by multiplying a ordinary conjugacy class $\mathscr{C}$ on the right by $w_{J}$. Fix $w \in \mathscr{C}_{F}$, and let $T_{w}$ be the maximal torus of $U(n)$ associated to $w$.

Theorem 3.2. Suppose $\sigma \in \hat{S}_{n}$. Then

$$
\begin{aligned}
R_{\sigma} & =\frac{1}{|W(T)|} \sum_{w \in W} \operatorname{tr}\left(\sigma\left(w w_{J}\right)\right) R_{w} \\
& =\frac{1}{|W(T)|} \sum_{\mathscr{C}}|\mathscr{C}| \operatorname{tr}(\sigma(w)) R_{w w_{J}}
\end{aligned}
$$

is a unipotent representation (up to sign) of $U(n)$, and all unipotent representations arise in this manner.

Define $\operatorname{sgn}(\sigma) \in\{+,-\}$ by the requirement that $\operatorname{sgn}(\sigma) R_{\sigma}$ have positive dimension.

Partitions $p=\left[p_{1}, p_{2}, \ldots, p_{j}\right] \quad\left(p_{i} \in \mathbf{N}, p_{i} \geq p_{i+1}, n=\sum p_{i}\right)$ of $n$ parametrize both conjugacy classes and irreducible representations of $S_{n}$. Indeed,

the conjugacy class $\mathscr{C}_{p}$ is the set of elements whose cycle lengths are the $p_{i}$ 's.

To describe the irreducible representation $\sigma_{p}$ associated to $p$, recall the Young diagram $D[p]$ attached to $p$ has $p_{i}$ blocks in its $i$ th row. Let $p^{t}=\left[p_{1}^{t}, p_{2}^{t}, \ldots\right.$, $\left.p_{j^{\prime}}^{t}\right]$ be the dual partition to $p$, i.e. the diagrams $D[p]$ and $D\left[p^{t}\right]$ are related by switching rows to columns and vice versa. Also, let

$$
I(p)=\operatorname{Ind}_{S_{p_{1}^{t}} \times S_{p_{2}^{t}} \times \cdots \times S_{p_{j^{\prime}}}} S .
$$

Then, $\sigma_{p}$ is characterized as

$\sigma_{p}$ is contained in $I(p)$ but is not contained in $I(q)$ for $q<p$. 
Here, $q<p$ is the parabolic ordering

$$
\sum_{i=1}^{k} q_{i}<\sum_{i=1}^{k} p_{i}
$$

For example, $[1,1, \ldots, 1]$ (resp. [n]) is a minimal (resp. maximal) element.

Theorem 3.3 [LS]. A unipotent representation $R_{\sigma}$ is cuspidal if and only if $\sigma$ corresponds to a partition $[k, k-1, \ldots, 1]$

In particular, $n=k(k+1) / 2$ must be a triangular number.

If $1 \leq k \leq n$, it is convenient for later use to consider $S_{n-k} \times S_{k} \subset S_{n}$. Let $p$ and $q$ be partitions of $(n-k)$ and $k$ respectively. We recall the LittlewoodRichardson rule which determines the constituents of

$$
I\left(\sigma_{p} \times \sigma_{q}\right)=\operatorname{Ind}_{S_{(n-k)} \times S_{k}}^{S_{n}} \sigma_{p} \otimes \sigma_{q} .
$$

For notational convenience, we shall use the dual partitions.

Littlewood-Richardson Rule. Suppose $q=\left[q_{1}, q_{2}, \ldots, q_{j}\right]$. Beginning with $i=$ 1 and continuing to $i=j$, add the blocks of the ith row of $D[q]$ (call them i-blocks) to $D[p]$ so that

(1) a Young diagram is obtained,

(2) no two i-blocks are in the same column,

(3) the rth block of row $(i+1)$ in $D[q]$ appears in a later row than the rth block of row $i$.

Let $Y=Y(p, q)$ be the collection (counted with multiplicity) of Young diagrams obtained. Then,

$$
I\left(\sigma_{p^{t}} \times \sigma_{q^{t}}\right)=\bigoplus_{\mu \in Y} \sigma_{\mu^{t}}
$$

As one application of this rule, fix a partition $p=\left[p_{1}, p_{2}, \ldots, p_{j}\right]$ of $n$ and for $1 \leq k \leq n$ define

$$
I\left(\sigma_{p}, S_{k}\right)=\frac{1}{\left|S_{k}\right|} \sum_{w^{\prime} \in S_{k}} \sigma_{p}\left(w^{\prime}\right) .
$$

Clearly, $I\left(\sigma_{p}, S_{k}\right)$ is the projection operator onto the $S_{k}$ fixed vectors of $\sigma$.

Proposition 3.4. If $j<k \leq n$, then $I\left(\sigma_{p}, S_{k}\right)=0$.

Proof. By Frobenius reciprocity, it is enough to show that $\sigma_{p}$ is not contained in $I\left(S_{k}\right)=\operatorname{Ind}_{1 \times S_{k}}^{S_{n}} 1$. Suppose $\sigma_{q} \subset I\left(S_{k}\right)$. By property (2) of the LittlewoodRichardson rule, the dual $D\left[q^{t}\right]$ has at least $k$ blocks in its first row. Since $D\left[p^{t}\right]$ has $j$ blocks in its first row, $\sigma_{p}$ cannot occur in $I\left(S_{k}\right)$.

We return to the general dual pair $\left(G, G^{\prime}\right)$ and review Srinivasan's work in [S] on the decomposition of the oscillator representation restricted to $\left(G, G^{\prime}\right)$. The results for unitary dual pairs, i.e. case (ii) are complete, while the case of $(S P, O)$, i.e. case (i), has a restriction. In each case we identify tori of $G$ with those of $G^{\prime}$ as in [S]. 
Case (ii). The representation $\omega_{m, n}$ of the dual pair $(U(m), U(n))$ decomposes as

$$
\begin{aligned}
(-1)^{m n} \omega_{m, n}=\sum_{k=0}^{\min (m, n)}(-1)^{k} & \sum_{(T) \subset U(k)} \frac{1}{|W(T)|} \\
& \cdot\left\{\sum_{\theta \in \hat{T}} R_{T \times U(m-k)}^{U(m)}(\theta \times 1) \otimes R_{T \times U(n-k)}^{U(n)}(\theta \times 1)\right\} .
\end{aligned}
$$

Case (i). Let $\mathscr{Z}$ be the center of $G$. Assume

(1) the orthogonal group $O$ is on an even dimension space $W$, say $\operatorname{dim}(W)=2 n$,

(2) $|\mathbf{F}|$ is large enough so that the character group of each maximal torus $T_{w}^{F} / \mathscr{Z}$ has at least two regular orbits under the Weyl group action.

Denote the dimension of $V$ by $2 m$. Let $\mathscr{U}$ be the subspace of class functions on $G=S p(V) \times S O(W)$ spanned by the $R_{T}^{G}(\theta)$ 's and let $\omega_{\mathscr{U}}$ be the projection of $\omega_{m, n}$, viewed as a character, onto $\mathscr{U}$. Note that the connected group $S O(W)$ is being used instead of the disconnected group $O(W)$. Define $\epsilon \in\{+,-\}$ by the requirement $S O^{\epsilon}(2 n)=S O(W)$.

Subcase (i.a), $m<n$.

$$
\omega_{\mathscr{U}}=\sum_{k=0}^{m} \sum_{(T) \subset S p(2 k)} \frac{1}{|W(T)|} \sum_{\theta \in \hat{T}} \epsilon \epsilon^{\prime} R_{T \times S p(2 m-2 k)}^{S p(2 m)}(\theta \times 1) \otimes R_{T \times S O^{\epsilon}(2 n)}^{S \sigma^{\prime}(2 n-2 k)}(\theta \times 1) .
$$

Subcase (i.b), $m \geq n$.

$$
\begin{aligned}
\omega_{\mathscr{U}}= & \sum_{k=0}^{n-1} \sum_{(T) \subset S p(2 k)} \frac{1}{|W(T)|} \sum_{\theta \in \hat{T}} \epsilon \epsilon^{\prime} R_{T \times S p(2 m-2 k)}^{S p(2 m)}(\theta \times 1) \otimes R_{T \times S O^{\epsilon^{\prime}}(2 n-2 k)}^{S O^{\prime}(2 n)}(\theta \times 1) \\
& +\sum^{\prime} \epsilon(-1)^{m+n} \frac{2}{|W(T)|} \sum_{\theta \in \hat{T}} R_{T \times S p(2 m-2 n)}^{S p(2 m)}(\theta \times 1) \otimes R_{T}^{S O^{\prime}(2 n)}(\theta)
\end{aligned}
$$

Here, each maximal torus $T \subset S p(2 k)$ defines [S] a unique $\epsilon^{\prime \prime}$ by the requirement that $T$ also lie in $S O^{\prime \prime}$. The factor $\epsilon^{\prime}$ is defined by $\epsilon \cdot \epsilon^{\prime} \cdot \epsilon^{\prime \prime}=1$. Finally, the sum $\sum^{\prime}$ is over conjugacy classes of maximal tori $T \subset S p(2 n)$ which also lie in $S O^{\epsilon}(2 n)$.

\section{Theorem 3.5.}

(1) Suppose the representation $\pi \otimes \pi^{\prime}$ of the dual pair $(U(m), U(n))$ occurs in $\omega_{m, n}$. Then $\pi$ is a unipotent representation of $U(m)$ if and only if $\pi^{\prime}$ is a unipotent representation of $U(n)$.

(2) Under the assumption (3.11), suppose the representation $\pi \otimes \pi^{\prime}$ of the dual pair $(S p(2 m), S O(W))$ occurs in $\omega_{m, n}$. Then $\pi$ is a unipotent representation of $S p(2 m)$ if and only if $\pi^{\prime}$ is a unipotent representation of $S O(W)$. 
Proof. Statement (1) follows from Proposition 3.4, and Srinivasan's formulas for $\omega_{m, n}$. The proof of (2) is only slightly more involved. We prove (2) by contradiction. We need to recall some basic facts. Denote $G \cdot G^{\prime}$ by $H$, and the character $R_{T}^{G}(\theta) \times R_{T^{\prime}}^{G^{\prime}}\left(\theta^{\prime}\right)$ by $R_{T \cdot T^{\prime}}^{H}\left(\theta \times \theta^{\prime}\right)$. These characters form an orthogonal basis for $\mathscr{U}$. The irreducible characters $\mathscr{E}(H)$ can be partitioned into geometric conjugacy classes [L1]

$$
\mathscr{E}(H)=\coprod_{s} \mathscr{E}(H,(s)),
$$

where $s=s_{G^{*}} \cdot s_{G^{*}}$ runs over the semisimple conjugacy classes of the dual group $H^{*}=G^{*} \cdot G^{\prime *}$. The irreducible constituents of $R=R_{T \cdot T^{\prime}}^{H}\left(\theta \times \theta^{\prime}\right)$ all belong to one geometric conjugacy class $\mathscr{E}(H,(s))$. Let $s=s(R)$ be the geometric conjugacy class associated to $R$. The projection $\mathscr{E}(H,(s))_{\mathscr{U}}$ of the characters $\mathscr{E}(H,(s))$ onto the space of uniform functions is spanned by the $R^{\prime} s$ with $s=s(R)$. If $\kappa$ is a positive combination of representations in $\mathscr{E}(H,(s))$, then it follows from the fact that the regular representation is uniform that

$\kappa_{\mathscr{U}}$ is nonzero.

Suppose $\tau=\pi \otimes \pi^{\prime}$ occurs in $\omega_{m, n}$ and that one of the factors is unipotent while the other is not. The geometric conjugacy class $s=s_{G^{*}} s_{G^{\prime *}}$ attached to $\tau$ would have exactly one of $s_{G^{*}}, s_{G^{*}}$ equal to the identity element. However, by (3.12), (3.13) and (3.15) this is a contradiction.

From Theorem 2.2 we immediately obtain the following theorem.

Theorem 3.6. In the setting of Theorem 3.5, suppose $\pi$ is a cuspidal unipotent representation of $G$, and $\pi$ does not occur in the representation correspondence for any pair $\left(G, G_{k}^{\prime}\right)$ for any $k<n$. Then $\pi^{\prime}$ is a cuspidal unipotent representation.

\section{4}

If $m=j(j+1) / 2$ is a triangular number, let $\sigma_{m}$ be the irreducible representation of $S_{m}$ associated to the partition $[j, j-1, \ldots, 1]$ and $\pi_{c u}(m)$ the cuspidal unipotent representation $R_{\sigma_{m}}$. In this section we prove the following result.

Theorem 4.1. If $m=j(j+1) / 2$, and $n=j(j-1) / 2$ or $n=(j+1)(j+2) / 2$, then the oscillator representation $\omega_{m, n}$ of the dual pair $(U(m), U(n))$ contains $\pi_{c u}(m) \otimes \pi_{c u}(n)$.

The proof depends on combining Theorem 3.3, (3.1), (3.10) and the LittlewoodRichardson rule. As a preliminary simplification, we observe that (3.10) is symmetrical in $m$ and $n$. Hence, it does not matter which unitary group we view as preserving the Hermitian or the skew-Hermitian form. In particular, it is convenient to assume $m=j(j+1) / 2$, and $n=j(j-1) / 2$.

Fix $r \in \mathbf{N}$ and $k \leq r \quad(k \in \mathbf{N} \cup\{0\})$. View $U(k) \times U(r-k)$ inside $U(r)$. If $p, q$ are partitions of $k,(r-k)$ respectively, let $T(p)$ and $T(q)$ be the maximal $F$-tori in $U(k)$ and $U(r-k)$ associated to $\mathscr{C}_{p}$ and $\mathscr{C}_{q}$. Then

$$
T(p, q)=T(p) \times T(q)
$$


is a maximal $F$-torus in $U(r)$. Formula (3.1) can be written as

$$
R_{T(p) \times U(r-k)}^{U(r)}(1)=\frac{1}{\left|S_{r-k}\right|} \sum_{\mathscr{C}_{q}}\left|\mathscr{C}_{q}\right| \cdot R_{T(p, q)}^{U(r)}(1) .
$$

In terms of the unipotent representations $R_{\sigma} \in \hat{U}(r)$, i.e. inversion of Theorem 3.2,

$$
R_{T(p, q)}^{U(r)}(1)=\sum_{\sigma \in \hat{S}_{r}} \operatorname{tr}(\sigma(p, q)) \cdot R_{\sigma}
$$

Here, $\operatorname{tr}(\sigma(p, q))$ is the character value of $\sigma$ on the conjugacy class in $S_{r}$ gotten by concatenating the partitions $p$ and $q$. Denote the multiplicity of $R_{\sigma}$ in $R_{T(p) \times U(r-k)}^{U(n)}(1)$ by $m(\sigma, p)$. Substitution of (4.3) into (4.1) yields

$$
m(\sigma, p)=\frac{1}{\left|S_{r-k}\right|} \sum_{\mathscr{C}_{q}}\left|\mathscr{C}_{q}\right| \cdot \operatorname{tr}(\sigma(p, q)) .
$$

This formula can be rewritten in a more useful fashion as follows. Consider $S_{k} \times S_{r-k} \subset S_{r}$. Let $w_{p} \in S_{k}$ have cycle lengths given by $p$. Then,

$$
\begin{aligned}
m(\sigma, p) & =\operatorname{tr}\left(\sigma\left(w_{p}\right) \cdot \frac{1}{\left|S_{r-k}\right|} \sum_{w^{\prime} \in S_{r-k}} \sigma\left(w^{\prime}\right)\right) \\
& =\operatorname{tr}\left(\sigma\left(w_{p}\right) \cdot I\left(\sigma, S_{r-k}\right)\right)
\end{aligned}
$$

where $I\left(\sigma, S_{r-k}\right)$ is the operator defined in (3.9).

Recall that we are assuming

(1) $m=j(j+1) / 2$ (resp. $n=j(j-1) / 2$ ),

(2) $\sigma_{m}\left(\right.$ resp. $\left.\sigma_{n}\right)$ is the irreducible representation of $S_{m}$ (resp. $S_{n}$ ) associated to the partition $[j, j-1, \ldots, 1]$ (resp. $[j-1, j-2, \ldots, 1])$.

As an immediate consequence of Proposition 3.4 we have

$$
I\left(\sigma_{m}, S_{m-k}\right)=0 \quad(k<n) .
$$

Theorem 4.1 is based on (4.6) and following proposition.

Proposition 4.2. For $k=n$, and $p$ a partition of $n$

$$
m\left(\sigma_{m}, p\right)=\operatorname{tr}\left(\sigma_{n}(p)\right)
$$

Proof. We consider the restriction of $\sigma_{m}$ to the subgroup $S_{n} \times S_{j}$. Fix a representation $\tau=\sigma_{a} \otimes \sigma_{[1, \ldots, 1]}$ of $S_{n} \times S_{j}$ trivial on $S_{j}$. By Frobenius reciprocity and the Littlewood-Richardson rule, $\tau$ occurs in the restriction if and only if $p^{t}=p$ lies in $Y\left(a^{t},[n]\right)$. By property (2) of the rule, $a$ must be the partition $[j-1, j-2, \ldots, 1]$. It follows that $m\left(\sigma_{m}, p\right)=\operatorname{tr}\left(\sigma_{n}(p)\right)$.

Proof of Theorem 4.1. Consider formula (3.10). For $k \neq n,(4.6)$ says that $\pi_{c u}(m)$ does not occur in $R_{T \times U(m-k)}^{U(m)}(1)$. The multiplicity of the representation $\pi_{c u}(m) \otimes \pi_{c u}(n)$ in $R_{T \times U(n)}^{U(m)}(1) \otimes R_{T}^{U(n)}(1)$ is $\operatorname{tr}\left(\sigma_{n}(p)\right) \cdot \operatorname{tr}\left(\sigma_{n}(p)\right)$. By the orthgonality relations for $S_{n}$ we conclude $\pi_{c u}(m) \otimes \pi_{c u}(n)$ occurs in $\omega_{m, n}$.

In this section we compute the cases of first occurrence for orthogonalsymplectic pairs. We begin by reviewing Lusztig's results [L2] on the cuspidal unipotent representations of orthogonal and symplectic groups. 
Theorem 5.1. The following groups:

(1) $S p(2 n), n=k(k+1)$,

(2) $S O^{\epsilon}(2 n), n=k^{2}, \epsilon=\operatorname{sgn}(-1)^{k}$,

(3) $S O(2 n+1), n=k(k+1)$

are the only groups in their respective Lie families which possess a cuspidal unipotent representation. In each case, the specified group $G$ has a unique cuspidal unipotent representation $\pi_{c u}$.

We have defined a unipotent representation of an orthogonal group $O^{\epsilon}$ to be a constituent of $\operatorname{Ind}_{S O^{\epsilon}}^{O^{\epsilon}}(\pi)$, where $\pi$ is a unipotent representation of $S O^{\epsilon}$. The uniqueness of $\pi_{c u}$ implies

$$
\operatorname{Ind}_{S O^{*}}^{O^{*}}\left(\pi_{c u}\right)=\pi_{c u}^{\alpha} \oplus \pi_{c u}^{\beta}
$$

where $\pi_{c u}^{\alpha}$ and $\pi_{c u}^{\beta}$ are irreducible cuspidal unipotent representations of $O^{\epsilon}$. We use the superscripts $\alpha$ and $\beta$ instead of \pm to avoid confusion with the notation distinguishing $O^{+}$from $O^{-}$. These representations differ by tensoring with the $s g n$ character of $O$.

Theorem 5.2. The representation correspondence $\hat{G} \rightarrow \hat{G}^{\prime}$ for the dual pair $\left(G, G^{\prime}\right)=\left(S p(2 m), O^{ \pm}(2 n)\right)$ takes the cuspidal unipotent representation of $G$ to a cuspidal unipotent representation of $G^{\prime}$ for $\left(G, G^{\prime}\right)$ as follows:

(1) $\left(\operatorname{Sp}(2 k(k+1)), O^{\epsilon}\left(2 k^{2}\right)\right)\left(\epsilon=\operatorname{sgn}\left((-1)^{k}\right)\right), \pi_{c u} \rightarrow \pi_{c u}^{\beta}$,

(2) $\left(\operatorname{Sp}(2 k(k+1)), O^{\epsilon}\left(2(k+1)^{2}\right)\right)\left(\epsilon=\operatorname{sgn}\left((-1)^{k+1}\right)\right), \pi_{c u} \rightarrow \pi_{c u}^{\alpha}$.

The following information may be read off of [H1]. Let $\omega_{j, \epsilon}(m)$ be the oscillator representation of the pair $S p(2 m) \times O^{\epsilon}(2 j)$ restricted to $S p(2 m)$.

\section{Proposition 5.3.}

(1) Every irreducible representation of $S p(2 m)$ is contained in the direct sum $\omega_{m,+}(m) \oplus \omega_{m,-}(m)$.

(2) The trivial representation of $S p(2 m)$ is contained in $\omega_{m+1,-}(m)$.

(3) $\omega_{j, \epsilon}(m) \otimes \omega_{k, \epsilon^{\prime}}(m) \simeq \omega_{j+k, \epsilon \epsilon^{\prime}}(m)$ for all $j, k, \epsilon, \epsilon^{\prime}$.

Proof of Theorem 5.2. Let $\pi_{c u}(m)$ denote the cuspidal unipotent representation of $G_{m}=S p(2 m)$. Let $\mathbf{N}_{e}$ and $\mathbf{N}_{o}$ denote the even and odd nonnegative integers respectively. The representations $\left\{\omega_{s,+}(m)\right\}_{s \in \mathbf{N}_{e}}$ (resp. $\left\{\omega_{t,-}(m)\right\}_{t \in \mathbf{N}_{o}}$ are representations of $S p(2 m)$ corresponding to the Witt series $\left\{O^{+}(2 s)\right\}$ (resp. $\left.\left\{O^{-}(2 t)\right\}\right)$. Fix $m=k(k+1)$, and consider the first occurrence of $\pi_{c u}(m)$ in $\left\{\omega_{s,+}(m)\right\}_{s}$ (resp. $\left\{\omega_{t,-}(m)\right\}_{t}$. Thus let $s$ (resp. $t$ ) be minimal such that $\pi_{c u}(m) \subset \omega_{s,+}(m)$ (resp. $\left.\pi_{c u}(m) \subset \omega_{t,-}(m)\right)$. By Proposition 5.3(1), either $s$ or $t$ is finite. Suppose for simplicity $s$ is finite. By Theorem 2.2 and Theorem 3.5 , the corresponding representation $\pi^{\prime}$ of $O^{+}(2 s)$ is a cuspidal unipotent representation, so $s=j^{2}$ for some $j \in \mathbf{N}_{e}$. Furthermore we have

$$
\begin{aligned}
\pi_{c u}(m) & \subset \omega_{s,+}(m) \simeq \omega_{s,+}(m) \otimes(\text { trivial }) \\
& \subset \omega_{s,+}(m) \otimes \omega_{m+1,-}(m) \quad \text { (by Proposition 5.3(2)) } \\
& \simeq \omega_{s+m+1,-}(m) \quad \text { (by Proposition 5.3(3)). }
\end{aligned}
$$

A similar argument holds if $t$ is finite, so we conclude both $s$ and $t$ are finite, and $t=j^{2}$ for some $j \in \mathbf{N}_{o}$. 
Thus we see that the cases of first occurrence establish two maps from the set of cuspidal unipotent representations $\pi_{c u}(k(k+1)), k \in \mathbf{N} \cup\{0\}$ of the symplectic groups, to the set of cuspidal unipotent representations of the split orthogonal groups $O^{+}\left(2 s^{2}\right), s \in \mathbf{N}_{e}$ (resp. $O^{-}\left(2 t^{2}\right), t \in \mathbf{N}_{o}$ ).

It remains only to compute the correspondences $k \rightarrow s$ and $k \rightarrow t$. We will see that they are both bijections. We simply start with $k=s=0, t=1$, and work our way up.
(a)
$S p(0)$
(trivial)
$\begin{array}{cc}\rightarrow & (\text { trivial }) \\ \rightarrow & (\text { trivial }) \\ \rightarrow & (\text { sgn }) \\ \rightarrow & \pi_{c u}^{\alpha}\end{array}$
$O^{+}(0)$
(b) $\quad S p(0)$
(trivial)
$O^{-}(2)$
(c) $\quad S p(4)$
$\pi_{c u}(2)$
(d) $\quad S p(4)$
$\pi_{c u}(2) \quad \rightarrow \quad \pi_{c u}^{\alpha}$
$O^{-}(2)$
$O^{+}(8)$
(e) $\operatorname{Sp}(2 k(k+1)) \quad \pi_{c u}(k(k+1)) \quad \rightarrow \quad \pi_{c u}^{\alpha} \quad O^{\epsilon}\left(2 k^{2}\right)$
(f) $S p(2 k(k+1)) \quad \pi_{c u}(k(k+1)) \quad \rightarrow \quad \pi_{c u}^{\beta} \quad O^{-\epsilon}\left(2(k+1)^{2}\right)$

We proceed to justify the entries in (5.3). Lines (a) and (b) are immediate. By Proposition 5.3(1), $\pi_{c u}(2)$ occurs in $\omega_{2,+}(2) \oplus \omega_{2,-}(2)$. Therefore $\pi_{c u}(2)$ must occur in $\omega_{0,+}(2) \oplus \omega_{1,-}(2)$ since this will correspond to the first occurrence of a representation of an orthogonal group, which is therefore cuspidal unipotent. But all the unipotent representations of $O^{+}(0)$ and $O^{-}(2)$ are accounted for already except for the sgn representation of $O^{-}(2)$. This proves (c).

Next we see that

$$
\begin{aligned}
\pi_{c u}(2) & \subset \omega_{1,-}(2) \otimes(\text { trivial }) \\
& \subset \omega_{1,-}(2) \otimes \omega_{3,-}(2) \quad \text { by Proposition } 5.3(2) \\
& \simeq \omega_{4,+}(2)
\end{aligned}
$$

Part (d) follows immediately from (5.4).

Repeating steps (c) and (d) (inductively) we see the cuspidal unipotent representation $\pi_{c u}$ of $\operatorname{Sp}(2 \mathrm{k}(\mathrm{k}+1))$ corresponds to cuspidal unipotent representations of $O^{\epsilon}\left(2 k^{2}\right)$ and $O^{-\epsilon}\left(2(k+1)^{2}\right)$ respectively $\left(\epsilon=(-1)^{k}\right.$ as usual). We label these representations $\pi_{c u}^{\alpha}$ and $\pi_{c u}^{\beta}$ respectively; this verifies (e) and (f).

Thus we obtain every cuspidal unipotent representation of the orthogonal groups exactly once. Note that we have used each cuspidal unipotent representation of each symplectic group twice, once for each Witt series of orthogonal groups. This completes the proof of the theorem.

We note that it follows from a converse to Theorem 2.4 (cf. [H1, Corollary 5.3.1] or [K, Theorem 2.1]) that outside of the case of first occurrence, cuspidal representations will not correspond. In particular, this means the cases listed in Theorem 4.1 and 5.2 are the only cases in which the cuspidal unipotent representations correspond.

\section{REFERENCES}

[A] J. Adams, L-functoriality for dual pairs, Astèrisque-Orbites Unipotentes et Représentations, 171-172 (1989), 85-129.

[C] R. Carter, Finite groups of Lie type: Conjugacy classes and complex characters, Wiley, New York, 1985.

[DL] P. Deligne and G. Lusztig, Representations of reductive groups over finite fields, Ann. of Math. 103 (1976), 103-161. 
[H1] R. Howe, Invariant theory and duality for classical groups over finite fields, preprint.

[H2] _,$L^{2}$-duality for stable reductive dual pairs, preprint.

[K] S. Kudla, On the local theta-correspondence, Invent. Math. 83 (1986), 229-255.

[L1] G. Lusztig, Characters of reductive groups over a finite field, Princeton Univ. Press, Princeton, N.J., 1984.

[L2] _ Irreducible representations of finite classical groups, Invent. Math. 43 (1977), 125-175.

[LS] G. Lusztig and B. Srinivasan, The characters of the finite unitary groups, J. Algebra 49 (1977), 167-171.

[MVW] C. Moeglin, M. F. Vignéras and J. L. Waldspurger, Correspondances de Howe sur un corps p-adique, Lecture Notes in Math., vol. 1291, Springer, Berlin, 1987.

[S] B. Srinivasan, Weil representations of classical groups, Invent. Math. 51 (1979), 143-153.

[V] D. Vogan, Unitary representations of reductive Lie groups, Princeton Univ. Press, Princeton, N.J., 1987.

Department of Mathematics, University of Maryland, College Park, Maryland 20742

E-mail address: jda@math.umd.edu

Department of Mathematics, University of Michigan, Ann Arbor, Michigan 48109

E-mail address: moy@math.lsa.umich.edu 